
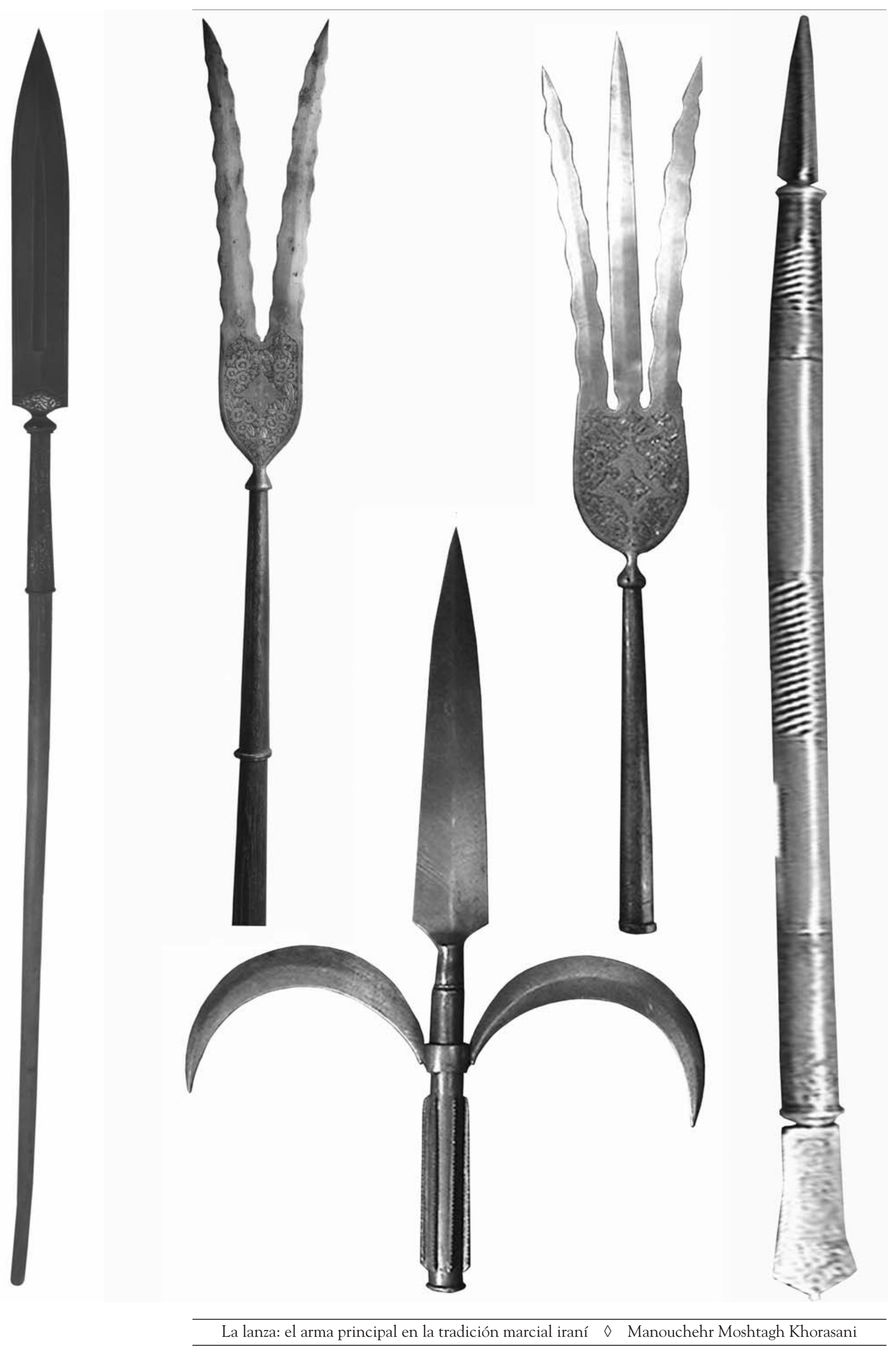


\title{
La lanza: \\ El arma principal en la tradición marcial iraní
}

\author{
MANOUCHEHR MOSHTAGH KHORASANI

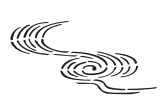

\begin{abstract}
Resumen
En la tradición marcial, las lanzas siempre han tenido un papel muy importante como armas principales en el campo de batalla, como ejemplifica la historia militar iraní. El alcance y poco coste de la lanza fueron los factores más importantes para que se creasen formaciones de soldados con lanzas. Además, las técnicas del combate con lanza eran relativamente sencillas y se podía aprender muy rápidamente en comparación con las técnicas de otras armas como las espadas. La primera parte de este artículo describe algunos tipos de lanzas que se utilizaron en el periodo islámico de Irán. A continuación se analizan las formas de portar la lanza, para a continuación detallar diversas técnicas de ataque, finta, ataque al animal que montaba el oponente y de defensa con lanza según las describen los manuscritos persas.
\end{abstract}

\section{Introducción}

Como arma compuesta, la lanza dispone de un asta (palo de madera, caña o metal) y una hoja afilada o puntiaguda en uno de sus extremos, que podía adoptar diversas formas. Las lanzas han jugado un papel muy importante en la historia militar de Irán, al igual que en la historia militar de muchos otros pueblos, gracias a aspectos tales como su efectividad, alcance y economía de fabricación. Las lanzas también eran utilizadas para la caza, y por ello se pueden encontrar abundantes ejemplos de lanzas en todas las regiones y periodos de la historia de Irán, como las lanzas de bronce halladas en Marlik (1200 a.C.) o en Luristán (1000 a.C.), respectivamente al norte y suroeste de Irán. La lanza tenía fama de ser la reina de las armas durante las batallas y podía utilizarse tanto a pie como a caballo para punzar y golpear a largo alcance. Así, en el ejército de la dinastía aqueménida (558-330 a.C.) los soldados que luchaban con lanza tenían un rango más alto (Pur Davood, 1969/1347:41), y el libro sagrado Zoroástrico Avesta también cita el papel principal de la lanza, a la que denomina aršti (Íbídem: 41, Bahrāmi y Joneydi, 1990/1369:125), palabra que también designa a la lanza en persa antiguo (vea la inscripción de Dāryuš Bozorg en Naqš-e Rostam DNa44).

Durante el periodo del imperio parto (250 a.C.-228 d.C.) la lanza mantuvo su importancia, especialmente en las unidades pesadas de caballería llamadas cataphract. Estos soldados tenían una armadura pesada que les protegía totalmente el cuerpo y

PÁGINA ANTERIOR: DE IZQUIERDA A DERECHA, UNA LANZA CON HOJA DE ACERO AL CRISOL DEL PERIODO SAFÁVIDA (15021722), PUNTA DE LANZA BIDENTE DEL PERIODO QĀJĀR (1794-1925), PUNTA DE LANZA CON UNA HOJA Y DOS GANCHOS DEL PERIODO ZAND (17501794), PUNTA DE LANZA TRIDENTE DEL PERIODO QĀJĀr (1794-1925), JABALINA DEL PERIODO AFŠĀRID (1737-1802).

Todas las fotografias son cortesía del autor, excepto cuando se señale lo contrario. 
luchaban con una lanza pesada que medía aproximadamente 2 metros; se decía que un caballero de la caballería pesada parta podía atravesar a dos soldados romanos de infantería con un solo golpe de lanza (Wilcox, 2001:10). Por su parte, la caballería ligera parta estaba armada con arcos y flechas (Zoka, 1971/1350:117), siendo el arco el arma más importante para las unidades partas, seguido inmediatamente por la lanza.

La tradición de usar caballería pesada continuó durante el periodo del imperio sasánida (241-651 d.C.). En esta época la palabra aršti, del persa antiguo, que designaba la lanza, se transformó en aršt, del persa medio (véase Farahvaši, 2002a/1381:535). Desde el principio del periodo, la caballería sasánida utilizó la lanza como arma prin-

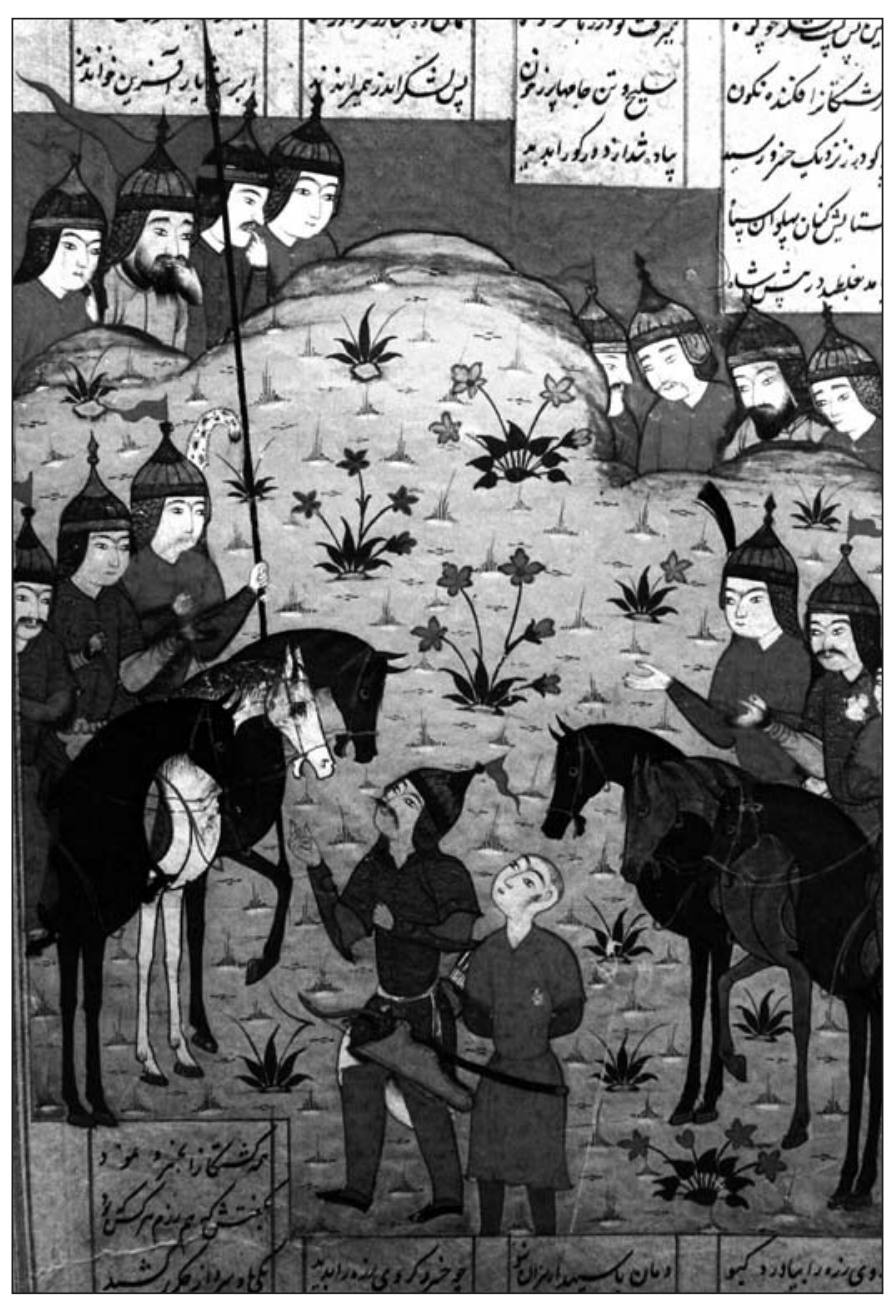
cipal (Farrokh, 2005:13), formando en fila durante las batallas. A su espalda se situaban los arqueros, en segunda fila, y los elefantes de guerra en tercera fila (Zoka, 1971/1350:117). Cuando la caballería pesada cargaba, los arqueros disparaban continuamente hacia las formaciones del enemigo, cubriendo de este modo la carga (Wiesehöfer, 1993:264).

Tras la conquista musulmán de Irán (651 d.C.), la lanza mantuvo su importancia como el arma principal de batalla. Los manuscritos persas citan diferentes tipos de lanzas y jabalinas que se utilizaban durante las mismas, e igualmente describen diferentes métodos para templar la hoja de lanza, como el manuscrito Bayān al-Sanā'àt (Taflisi, 1975/1354:339), que describe que para templar una hoja de lanza que causa heridas negras -esto es, graves- y mate al enemigo, se tienen que tomar huevos de avispas y abejas y quemarlos, para después mezclar el producto resultante con sangre de burro, agua, y llevarlo a ebullición. Después la hoja de lanza se templa en este líquido para lograr el mortífero resultado. Los manuscritos persas también describen otros detalles, como el manuscrito $\bar{A} d \bar{a} b$ alHarb va al-Šojā-e (Mobārak Šāh Faxr-e Modabbar, 1967/1346:261), que señala que para el asta de lanza se usaba la madera de sauce, o el Dārābnāme (Beiqami, 2002/1381:623; vol.1), que cita que también se utilizaba acero hueco para el asta.

MINIATURA DE UN MANUSCRITO DE $\check{S} \bar{A} H N \bar{A} M E-Y E \check{S} \bar{A} H$ MAHMUD, DEL S. XVI, MOSTRANDO A UN CABALLERO ARMADO CON UNA LANZA LARGA DE CABALLERÍA.

Cortesía de la Sepehsālār Library.
La lanza y sus tipologías

Existen doce palabras en persa que designan la lanza:

a) Neyze: es el término genérico para designar la lanza en persa. Se utiliza para designar tanto la pica que utilizaba la infantería como la lanza larga (kontos) de la caballería ${ }^{1}$. Otra palabra para referirse a la lanza en un sentido genérico es mexras (Lexicón Digital de Dehxodā).

b) Senān: Normalmente la palabra senān significa la punta de la lanza; no obstante algunos manuscritos persas utilizan esta palabra para referirse a lanza en general, como las epopeyas Šāhnāme, del s. X (Ferdǒsi, 1995/1384:190) y Garšāsbnāme, del s. XI (Asadi Tusi, 1938:1317:5)². 
c) Šel: se trata de una jabalina (lanza arrojadiza) con una cabeza bidente (en forma de media luna) o en forma de tridente. Los guerreros portaban cinco y hasta diez šel para lanzarlas a sus enemigos (Ma'tufi, 1999/1378:391) 3. Mobārak Šāh Faxr-e Modabbar (1967/1346:260) explica que šel es arma característica de hindúes y afganos.

d) Xešt: es una lanza corta que tiene un anillo en su mitad, al que se ataba una cuerda, que por su otro extremo se ataba a la mano. De este modo, tras el lanzamiento se podía recuperar la lanza tirando de la cuerda (véase Ma'tufi, 1999/1378:390; Manučehri Dāmqāni, 1984/1363:179)4.

e) Zubin: es la jabalina que se utilizaba habitualmente en la batalla, como refieren las epopeyas Šāhnāme, del s. X (Ferdŏsi, 1995/1384:313), Romuz-e Hamze, recolectado en el s. XV (1940/1359 Hégira:97) y Šahanšahnāme, del s. XIX (Fathali Xān Sabā, 11) $)^{5}$. Otra palabra para designar la jabalina en persa es harbe, como describen los manuscritos Tārix-e Beihaqi, del s. XI (Beihaqi, 2004/1383:137) y $\bar{A} d \bar{a} b$ alHarb va al-Šojā-e, del s. XII (Mobārak Šāh Faxr-e Modabbar, 1967/1346:330)'.

f) Durbāš: es una lanza con una cabeza de dos dientes que estaba decorada con joyas y se portaba por delante del rey para que la gente se mantuviese a distancia; es decir, el durbāš servía como una señal para que la gente no se acercase al rey. Nótese que dur significa "lejano, distante" y $b \bar{a} \breve{s}$ procede del verbo budan (ser). En el manuscrito $\bar{A} d \bar{a} b$ al-Harb va al-Šojā-e, del s. XII (Mobārak Šāh Faxr-e Modabbar, 1967/1346:260), se señala que los guardaespaldas del rey utilizaban un arma que se llamaba durbāš. Esta palabra también se encuentra en los tomos de poemas Manteq alTeyr (Attār Neišāburi, 1993/1372:54) y Masnavi Ma'navi, del s. XIII (Molavi, 2006/1385:713).

g) Cangāl: es el tridente, citado en los tomos de poemas Divān-e Mas'ud Sa'd Salmān, del s. XI (Sa'd Salmān, 1995/1374:308) y Šahanšahnāme, del s. XIX (Sabā, s.f: 185).

h) Jarid: esta palabra se utilizaba para designar un tipo de lanza y también de jabalina, pero de un modo más frecuente en este segundo sentido, como en los manuscritos Xold-e Barin (Qazvini Esfahāni, 2003/1382:453) y Rostam al Tavārix, del s. XIX (Āsef, 2003/1382:269-270).

i) Mezrāb o mezrāq: es un tipo de lanza corta normalmente con una cabeza de dos o tres dientes?

j) Remh: esta palabra se utilizaba para designar un tipo de lanza y/o jabalina ${ }^{8}$.

\section{Portar la lanza}

Existían diferentes formas de portar la lanza, como describen los manuscritos persas. El manuscrito Samak Ayyār del s. XI (al-Kāteb al-Rajāni, 2004/1383:503) describe la manera neyze bar duš (la lanza sobre el hombro), en la que el asta de la lanza se apoyaba sobre el hombro. Otra forma era aguantar el asta de lanza paralela a la oreja del caballo, como describen los manuscritos Majma' al-Ansāb, del s. XIV (Šabānkāre'i, 2002/1381:274), con la expresión neyze bar guš asb rāst kardan (recti- 
ficar la lanza a la oreja del caballo) y el manuscrito Samak Ayyār, del s. XI (al-Kāteb al-Rajāni, 2004/1383:435), con la expresión neyze rā be movāzāt guš asb gereftan vā be suy havāle kardan (coger la lanza paralela a la oreja del caballo y atacar). En caso de lanzas pesadas de caballería, se apoyaba la contera sobre el suelo y se tiraba de ella para compensar su peso; esto se realizaba en distancias cortas o directamente antes de la batalla, como describe el manuscrito Majma' al-Ansāb, del s. XIV (Šabānkāre'i, 2002/1381:274), con la expresión bon-e neyze dar xāk kešidan (apoyar la contera de la lanza sobre el suelo).

\begin{abstract}
IZQUIERDA:
Miniatura "HAZRAT-E AMIR EN BATALLA CONTRA EL EJÉRCITO DE TAHMASP ŠĀH”, DE UN MANUSCRITO DE XĀVARĀNĀME, DEL S. XV, EN LA QUE SE MUESTRA A UN GUERRERO (A LA DERECHA) QUE ESTÁ PORTANDO SU LANZA DEL MODO NEYZE BAR DUŠ (LA LANZA SOBRE EL HOMBRO).
\end{abstract}

Cortesia del Ministry of Guidance and Islamic Culture.

DERECHA:

DETALLE DE LA MINIATURA "BAHRĀM ČUBIN MATA A SĀVEH ŠĀH EN BATALlA”, DE UN MANUSCRITO DE Š $\bar{A} H N \bar{A} M E-Y E$ TAHMĀSPI (944 HÉGIRA/1537 D.C.), MUESTRA A BAHRĀM APLICANDO LA TÉCNICA NEYZE BAR GUŠ ASB RĀST KARDAN (RECTIFICAR LA LANZA A LA OREJA DEL CABALLO) ANTES DEL ATAQUE. VÉASE LA MINIATURA COMPLETA EN LA CONTRAPORTADA DE ESTE NÚMERO DE RAMA.

Cortesía del Rezā Abbāsi Museum.

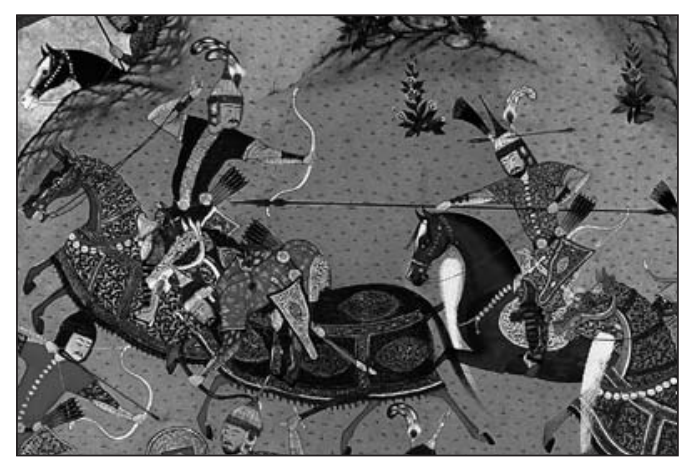

Técnicas de ataque con la lanza

Existen expresiones persas genéricas que designan el ataque con la lanza, como neyze āxtan (golpear con la lanza) y neyze afkandan y neyze andāxtan (lanzar la lanza), tal y como indica el Lexicón de Dehxodā, y la expresión neyze zadan (golpear con la lanza) en el manuscrito Garšāsbnāme, del s. XI (Asadi Tusi, 1938/1317:86) ${ }^{9}$. Para dar más fuerza a las lanzadas, a veces se giraba la lanza sobre la cabeza, como describe el manuscrito Dārābnāme, del s. XV (Beiqami, 2002/1381:97; vol.1), con la expresión neyze bar sar-e čang gardān kardan (girar la lanza sobre la palma [de las manos]). Otras técnicas específicas de ataque con la lanza son:

\section{Ataques a la cabeza con la lanZA}

La cabeza era un blanco favorito para asestar una lanzada, como refiere el manuscrito Abu Moslemnāme, del s. X (Tartusi, 2001/1380:82; vol. 2), con la expresión neyze havāle-ye sar kardan (atacar la cabeza con la lanza). Los manuscritos también citan diversos objetivos particulares en esta zona, como los ojos (manuscrito Abu Moslemnāme, del s. X, en Tartusi, 2001/1380:318; vol. 2), con la expresión neyze bar češm zadan (golpear el ojo con la lanza), el cuello (manuscrito Tārix-e Beihaqi, del s. XI, en Beihaqi, 2004/1383:588), con la expresión neyze bar galu zadan (golpear el cuello con la lanza), la garganta (manuscrito Abu Moslemnāme, del s. X, en Tartusi, 2001/1380:430; vol. 3), con la expresión neyze dar halq zadan (golpear la garganta con la lanza), la boca (manuscrito Dārābnāme, del s. XV, en Beiqami, 2002/1381:30; vol.1), con la expresión neyze bar dahan zadan (golpear la boca con la lanza), o la cara, (manuscrito $\mathrm{Abu}$ Moslemnāme, del s. X, en Tartusi, 2001/1380:73; vol. 4), con la expresión neyze bar surat zadan (golpear la cara con la lanza).

2. ATACAR LOS BRAZOS Y LOS HOMBROS CON LA LANZA

Como objetivos dentro de los brazos, los manuscritos persas citan las manos, como describe el manuscrito Zafarnāme, del s. XV (Yazdi, 1957/1336a:177), con la expre-

La lanza: el arma principal en la tradición marcial iraní $\diamond$ Manouchehr Moshtagh Khorasani 


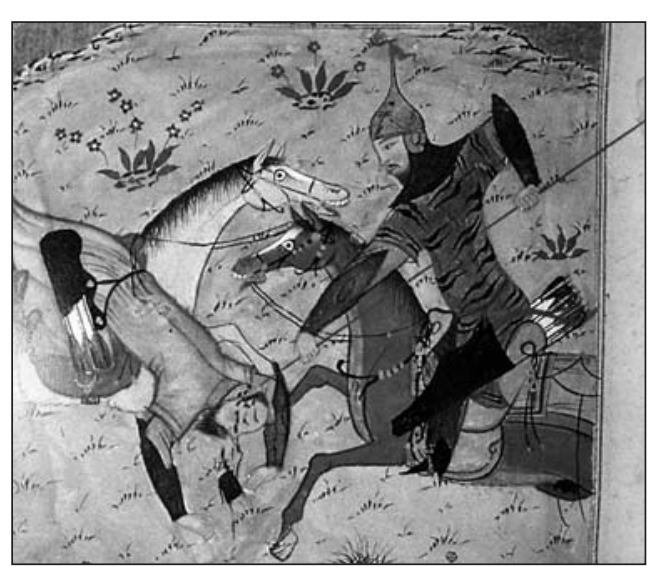

LA MINIATURA “LA LUCHA DE ROSTAM CON ALKUS, COMANDANTE DE TURÁN”, DE UN MANUSCRITO DE ŠĀHNĀME DEL S. XV, MUESTRA A ROSTAM ASESTANDO UNA LANZADA A LA CABEZA DE ALKUS APLICANDO LA TÉCNICA "ATACAR LA CABEZA CON LA LANZA". Cortesía de la National Library of Malek.

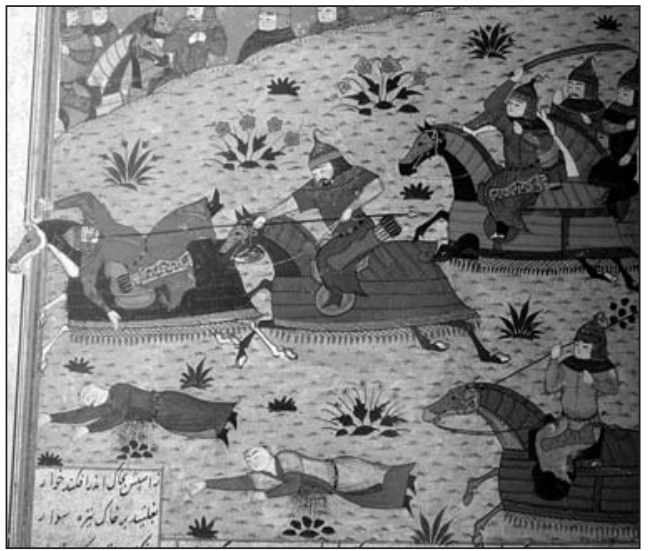

LA MINIATURA “ABU AL-MIHJAN EN LA BATALLA”, DE UN MANUSCRITO DE X'̄ VARĀNĀME DEL S. XV, MUESTRA AL GUERRERO DE LA DERECHA UTILIZANDO LA TÉCNICA NEYZE BAR ČEŠM ZADAN (GOLPEAR EL OJO CON LA LANZA). Cortesia del Ministry of Guidance and Islamic Culture.

sión neyze be dast zadan (golpear la mano con la lanza). También se atacaban los hombros, como refiere el manuscrito Abu Moslemnāme, del s. X (Tartusi, 2001/1380:113; vol. 4), con la expresión neyze bar ketf zadan (golpear el hombro con la lanza).

3. ATACAR EL PECHO CON LA LANZA

Otro blanco para un golpe de lanza era el pecho, como refiere el tomo de poemas Šāhnāme-ye Nāderi (Nāderi, 1968/1346:289), con la expresión neyze be sine furu kardan (perforar el pecho con la lanza). Existen otras expresiones para designar esta técnica, como neyze bar sine zadan (golpear el pecho con la lanza), en el manuscrito Romuz-e Hamze, recolectado en el s. XV (1940/1359 Hégira: 544), y neyze rā havāleye sine nemudan (enviar la lanza al pecho), en el manuscrito Širuye Nāmdār (2005/1384:199).

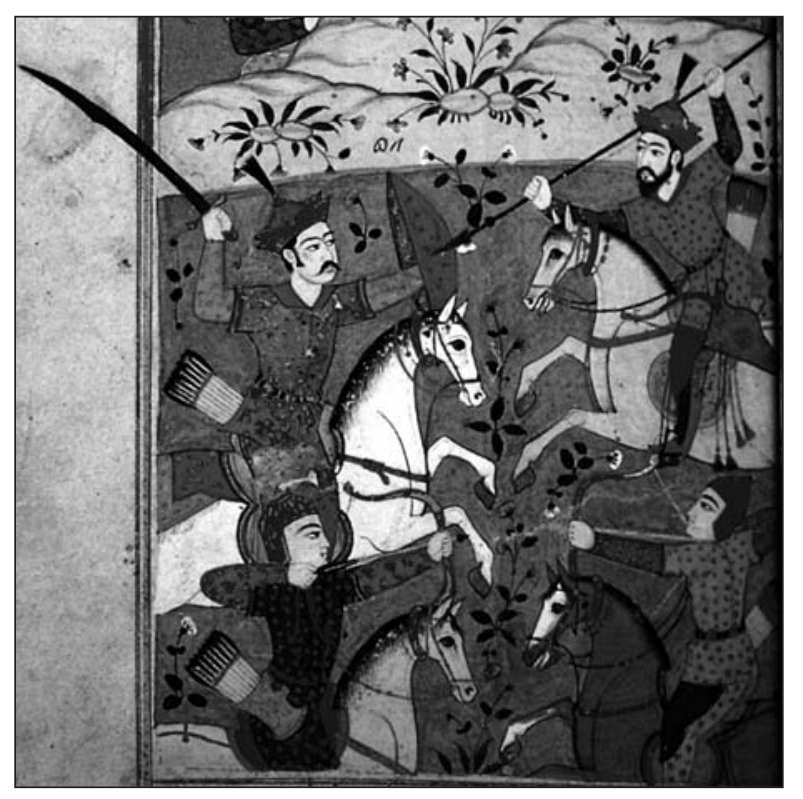

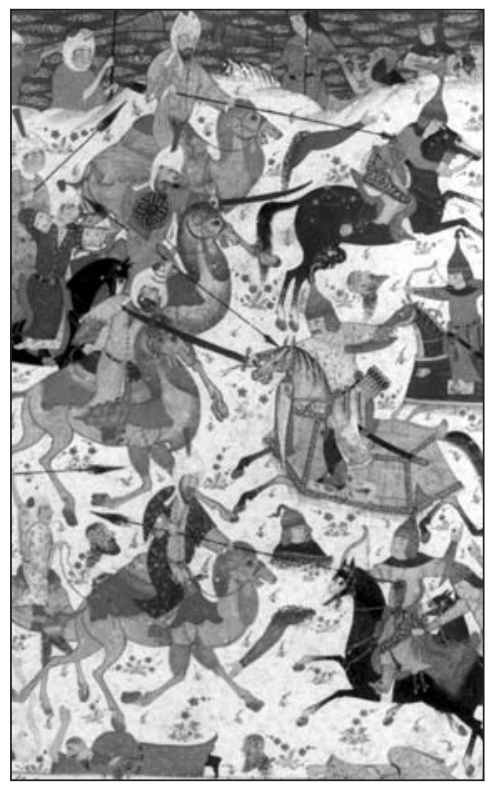

Revista de Artes Marciales Asiáticas $\diamond$ Volumen 4 Número 4 (70-85) - 2009
ABAJO IZQUIERDA:

MiniATURA "LA BATALLA DEL EJÉRCITO DE BIJAN CON YAZDGERD”, DE UN MANUSCRITO DE Š́̄HNĀME DEL S. XVI, EN LA QUE SE MUESTRA AL GUERRERO DE LA DERECHA UTILIZANDO LA TÉCNICA “ATACAR EL PECHO CON LA LANZA". Cortesia de la Library of Majles.

ABAJO DERECHA:

LA MINIATURA "LA BATALLA ENTRE DĀRĀB Y ŠO'AYB EL ÁRABE", DE UN MANUSCRITO DE $\check{S} \bar{A} H N \bar{A} M E$ DE XVI D.C., MUESTRA TRES TÉCNICAS CON LA LANZA (DE ARRIBA A ABAJO): "ATACAR LA ESPALDA CON LANZA", "ATACAR LA MANO CON LANZA" Y "ATACAR EL PECHO CON LANZA". Cortesía del Rezā Abbāsi Museum. 
1: LA MINIATURA "LA LUCHA DE ROSTAM FARROX HORMOZD CON SA`D BEN WAQQĀS Y EL ASESINATO DE ROSTAM DURANTE EL DOMINIO DE YAZDGERD", DE UN MANUSCRITO DE $\check{S} \bar{A} H N \overline{A M E}$ DEL S. XV, MUESTRA A AMBOS GUERREROS USANDO LA TÉCNICA “ATACAR EL ABDOMEN CON LA LANZA".

Cortesía de National

Museum of Iran.

2: LA MINIATURA "LA DERROTA DEL EJÉRCITO DEL REY DEL OESTE", DE UN MA-

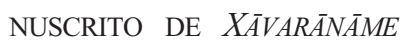
DEL S. XV, MUESTRA AL GUERRERO DE LA DERECHA UTILIZANDO LA TÉCNICA NEYZE BAR PAHLU ZADAN (GOLPEAR EL LADO DEL CUERPO CON LA LANZA).

Cortesia del Ministry of Guidance and Islamic

Culture

3: Detalle DE LA MiNiATURA "ROSTAM MATA KAHĀR KĀŠĀNI”, DE UN MANUSCRITO DE $\check{S} \bar{A} H N \bar{A} M E-Y E \check{S}_{\bar{A}} H$ TAHMĀSPI DATADO EN EL 1537 D.C., MUESTRA A DOS JINETES, ENTRE ELLOS ROSTAM (ABAJO), CLAVANDO UNA LANZA A SU OPONENTE EN LA ESPALDA.

Cortesía del Rezā Abbāsi Museum.

4: LA MINIATURA "LA BATALLA ENTRE ROSTAM Y QOLUN", DE UN MANUSCRITO DE $\check{S} \bar{A} H N \bar{A} M E$, MUESTRA A ROSTAM DERRIBANDO A SU OPONENTE TRAS CLAVARLE LA LANZA EN LA ESPALDA.

Cortesía del National Museum of Iran.

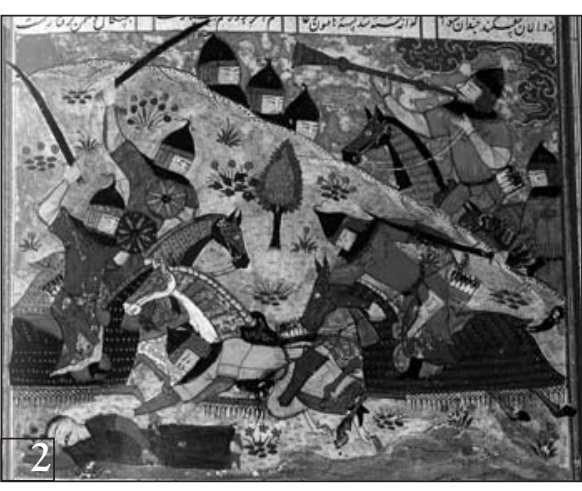

6. ATACAR LA ESPALDA CON LA LANZA

La espalda del oponente era un blanco fácil para una lanzada tanto en la batalla como cuando el enemigo se giraba para escapar o huía, como describe el manuscrito Majma' al-Ansāb, del s. XIV (Šabānkāre'i, 2002/1381:315), con la expresión neyze bar pošt zadan (golpear la espalda con la lanza).
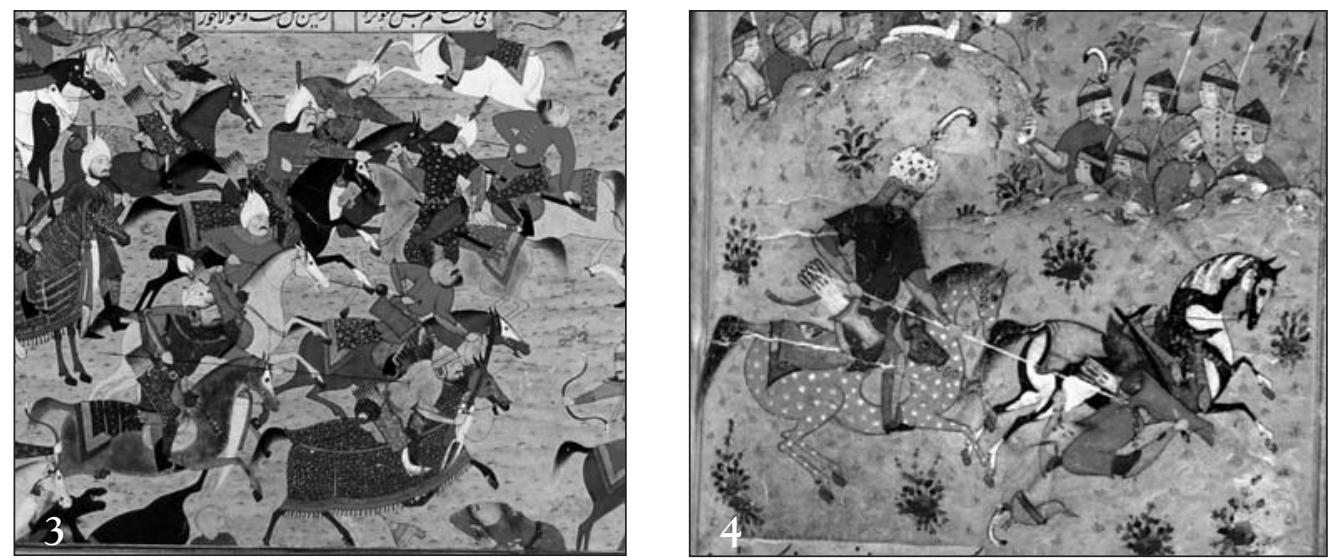

La lanza: el arma principal en la tradición marcial iraní $\diamond$ Manouchehr Moshtagh Khorasani 


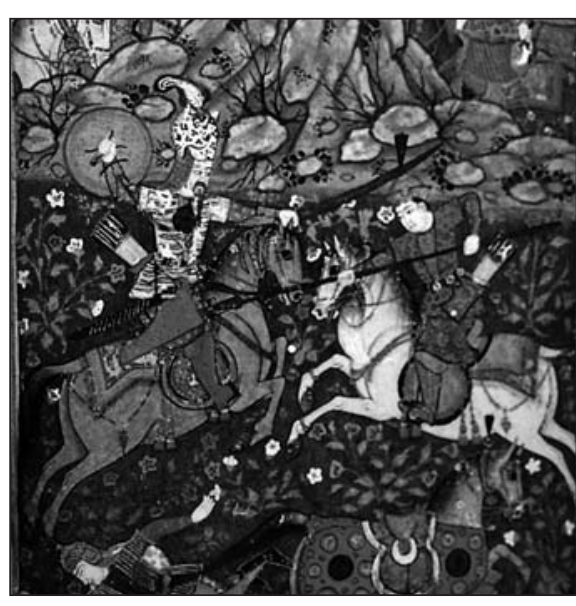

LA MINIATURA "MANUČEHR ATACA EL EJÉRCITO DE TUR”, DE UN MANUSCRITO DE $\check{S} \bar{A} H N \bar{A} M E$ DEL S. XVI, MUESTRA A UN GUERRERO EMPLEANDO LA TÉCNICA "GOLPEAR LA REGION INGUINAL CON LA LANZA".

Cortesía de la Library of Majles.

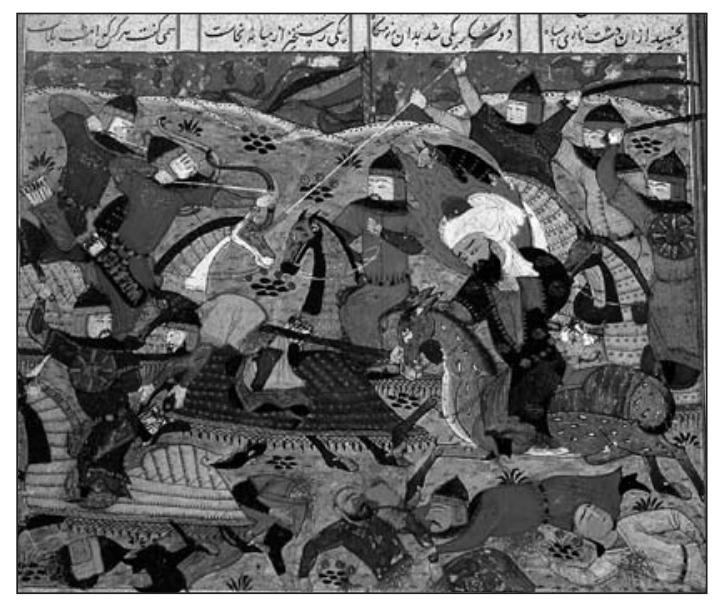

LA MiNIATURA “IMĀN Ali EN LA BATALLA" DE

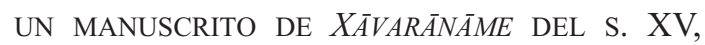
MUESTRA AL GUERRERO DE LA PARTE SUPERIOR UTILIZANDO LA TÉCNICA BON-E NEYZE ZADAN (GOLPEAR CON LA CONTERA DE LA LANZA). Cortesia del Ministry of Guidance and Islamic Culture.

7. ATACAR LA REGIÒN INGUINAL CON LA LANZA

Puesto que los jinetes lógicamente tenían las piernas abiertas al cabalgar sobre su montura, la región inguinal se mostraba como un buen blanco para asestar una lanzada, como describe el manuscrito Abu Moslemnāme, del s. X (Tartusi, 2001/1380:422; vol. 2), con la expresión neyze bar tohigāh zadan (golpear la region inguinal con la lanza).

8. ATACAR Al Oponente CON la CONTERA DE LA LANZA

La contera (extremo contrario a la punta de la lanza) podía utilizarse para golpear al oponente, como describe el manuscrito Šăhnāme, del s. X (Ferdŏsi, 1995/1384:190), con la expresión bon-e neyze zadan (golpear con la parte baja de la lanza).

9. CORTAR LAS CUERDAS DE LA ARMADURA DEL OPONENTE

Cuando la armadura era muy pesada y fuerte como para ser penetrada por las lanzadas, los manuscritos citan una técnica que consistía en cortar las cuerdas de la armadura del oponente para seguidamente continuar con otros ataques, como refiere el manuscrito Samak Ayyār, del s. XI (al-Kāteb al-Rajāni, 2004/1383:423), con la expresión band-e zereh rā boridan (cortar las cuerdas de la armadura).
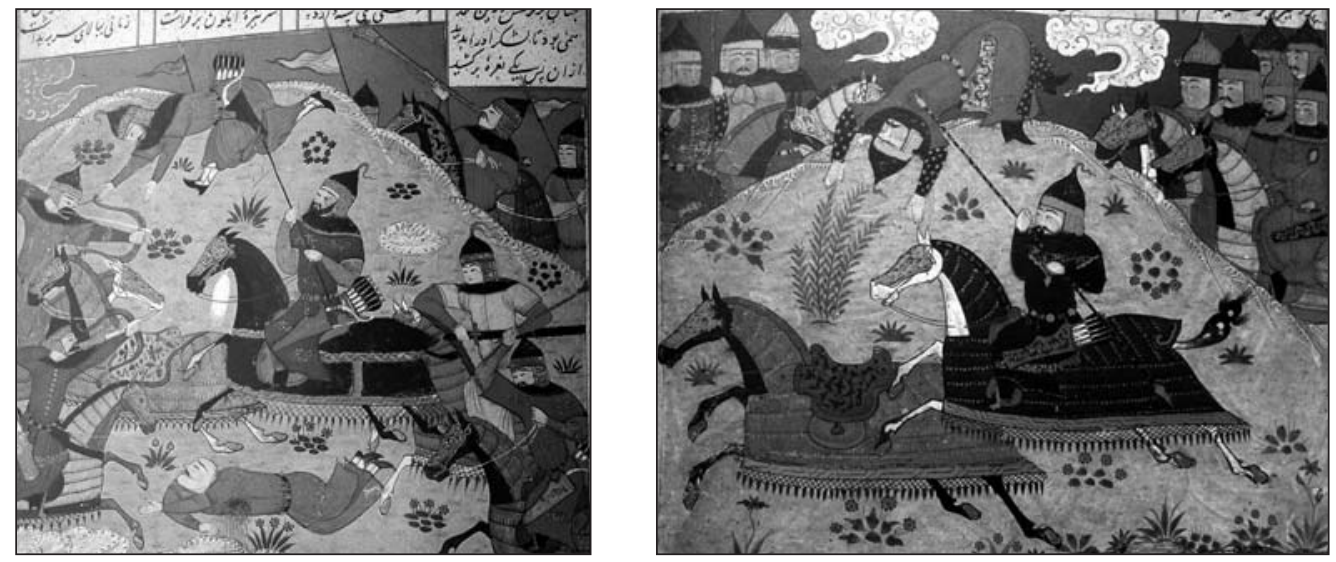

Revista de Artes Marciales Asiáticas $\diamond$ Volumen 4 Número 4 (70-85) - 2009

ABAJO IZQUIERDA:

LA MINIATURA “ABU ALI AL-MIHJAN MATA A SU OPONENTE CON UNA LANZA", DE UN MANUSCRITO

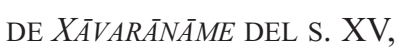
MUESTRA LA TÉCNICA $B E$ NEYZE AZ POŠT-E ASB BARGEREFTAN VA BAR ZAMIN ZADAN (TOMAR DEL CABALLO CON LA LANZA Y LANZARLO AL SUELO).

ABAJO DERECHA:

LA MINIATURA "SA D MATA A SU OPONENTE CON UNA LANZA", DE UN MANUSCRI-

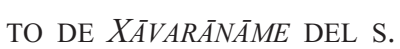
XV, MUESTRA LA TÉCNICA BE NEYZE AZ POŠT-E ASB BARGEREFTAN VA BAR ZAMIN ZADAN (TOMAR DEL CABALLO CON LA LANZA Y LANZARLO AL SUELO).

Cortesia del Ministry of Guidance and Islamic Culture. 


\section{GOLPEAR AL OPONENTE CON LA LANZA Y LANZARLO AL SUELO}

Esta técnica se basaba en empujar al oponente con la lanza, levantándolo con fuerza para lanzarlo al suelo a continuación, como refiere el manuscrito Majma' al-Ansāb (Šabānkāre'i, 2002/1381:265) con la expresión be neyze az pošt-e asb bargereftan va bar zamin zadan (tomar del caballo con la lanza y lanzarlo al sue1o), y el manuscrito Ādāb al-Harb va al-Šojā-e (Mobārak Šāh Faxr-e Modabbar, 1967/1346:261) con la expresión az zin robudan (robar la silla de montar).

\section{Técnicas de finta con la lanza}

Estas técnicas son técnicas de ataque en las que en primer lugar se fintaba un determinado golpe con la lanza, y cuando el oponente reaccionaba para defenderse se cambiaba la técnica sobre la marcha para ejecutar otra.

1. Mostrar la lanZa A LA IZQUiERDA Y GOLPEAR LA MANO A LA DERECHA El manuscrito Širuye Nāmdār (2005/1384:331) explica esta técnica con la expresión neyze az čap nešān dādan va az rāst bar band-e dast zadan (mostrar la lanza a la izquierda y golpear la mano a la derecha).

2. Mostrar la lanZa A LA IZQUiERDA y GOLPEAR EL ASTA DE LA LANZA [DEL OPONENTE] DESDE ABAJO

Se aplicaba esta técnica fintando un ataque de la izquierda, para rápidamente golpear el asta de la lanza del oponente desde abajo con el objeto de abrir un hueco en su guardia o desarmarlo, como describe el manuscrito Romuz-e Hamze, recolectado en el s. XV (1940/1359 Hégira: 60, 81), con la expresión neyze čap nemudan va az rāst be zir-e neyze zadan (mostrar la lanza a la izquierda y golpear el asta de la lanza desde abajo).

3. GIRAR LA LANZA Y CLAVAR LA PUNTA DE LA LANZA

Esta técnica consiste en girar la lanza sobre la cabeza o al frente del cuerpo y después asestar una lanzada a la espalda del oponente [que está escapando] con la punta de la lanza, como describe el manuscrito Dārābnāme, del s. XV (Beiqami, 2002/1381:385; vol.1), con la expresión neyze rā gardāndan va ta'n neyze bar kamargāh zadan (girar la lanza y golpear la espalda con la punta de la lanza).

\section{Técnicas de ataque al animal que montaba el oponente}

También se utilizaba la lanza para atacar al animal que montaba el oponente. Algunas de estas técnicas se presentan en los manuscritos persas como se explica a continuación.

\section{ATACAR LA FRENTE DEL CABALLO CON LA LANZA}

Como elemento más adelantado, a veces se utilizaba la lanza para atacar la frente del caballo que montaba el adversario, como indica el manuscrito Širuye Nāmdār (2005/1384:130) con la expresión neyze bar pišāni-ye markab zadan (golpear la frente del caballo con la lanza).

2. ATACAR EL PECHO DEL CABALlO CON LA LANZA

Otro blanco para atacar al caballo del oponente era el pecho, como explica el manuscrito Joneydnāme (Kufi, 1991/1380:221, traducido al turco en el año 914 de la Hégira - 1508 d.C.) con la expresión neyze bar sine-ye asb rāndan (atacar el pecho del caballo con la lanza). 


\section{ATACAR LA FRENTE DEL ELEFANTE CON LA LANZA}

La frente del elefante era otro blanco para atacar con la lanza, como indica el manuscrito Abu Moslemnāme, del s. X (Tartusi, 2001/1380:103; vol. 4), con la expresión neyze bar pišāni-ye pil zadan (atacar la frente del elefante con la lanza).

\section{Técnicas de defensa con la lanza}

Se utilizaba el asta de la lanza para defenderse de las lanzadas o de los ataques con otras armas del enemigo, como indican las siguientes expresiones:

\section{GOlPEAR ASTA CONTRA ASTA}

Con esta técnica se golpeaba el asta de la propia lanza contra el asta de la lanza del enemigo, como refieren los manuscritos Eskandarnāme, basado en relatos del s. XI y colectado en el s. XVII (Hakim, sin fecha:347), con la expresión neyze rā be neyze zadan (golpear la lanza contra la lanza) y Samak Ayyār, del s. XI (al-Kāteb al-Rajāni, 2004/1383:437), con la expresión neyze bar neyze andāxtan (meter la lanza sobre la lanza).

2. DefENDER La LANZADA DEL OPONENTE GOLPEANDO EL ASTA DE SU LANZA CON LA PROPIA ASTA

Esta técnica consiste en golpear con el asta de la propia lanza sobre el asta de la lanza del oponente, como indica el manuscrito Dārābnāme-ye Tarsusi (Tartusi, 1977/2536:124, vol. 2) con la expresión be čub neyze rā rad kardan (evadir con el palo [asta]).

3. DEFENDER LA LANZADA DEL OPONENTE GOLPEANDO BAJO SU ASTA CON LA PROPIA ASTA

Para defenderse también se golpeaba el asta de la propia lanza bajo el asta de la lanza del oponente, como explica el manuscrito Širuye Nāmdār (2005/1384:81) con la expresión neyze bar zir-e neyze zadan (golpear la lanza bajo la lanza).

4. SACAR El ASTA DE LA LANZA DEL OPONENTE DE SU MANO

En esta técnica se cogía el asta de la lanza del oponente con las propias manos y se le arrebataba con fuerza, como refieren los manuscritos Romuz-e Hamze, recolectado en el s. XV (1940/1359 Hégira:427), con la expresión neyze az kaf robudan (robar la lanza de mano) y Eskandarnāme, basado en relatos del s. XI y colectado en el s. XVII (Hakim, sin fecha: 533), con la expresión neyze rā az kaf birun āvardan (sacar la lanza de la palma de la mano).

5. AgarRAR El ASTA DE LA LANZA DEL OPONENTE

Una técnica de defensa contra los golpes de lanza del oponente era agarrar el asta de lanza del oponente y después atacarlo con su propia lanza, como explica el manuscrito Zafarnāme, del s. XV (Yazdi, 1957/1336a:177), con la expresión neyze rā gereftan (agarrar la lanza).

6. COGER El ASTA DE LANZA DEL OPONENTE BAJO LA AXILA Y DESARMARLO

Esta técnica consiste en coger el asta de la lanza del oponente bajo la axila y desarmarlo, como refiere el manuscrito Dārābnāme-ye Tarsusi (Tarsusi, 1977/2536;2324, vol. 1) con la expresión neyze zir-e baqal gereftan va az dast birun kardan (coger la lanza bajo la axila y sacar la lanza). 


\section{Conclusión}

La lanza fue un arma muy utilizada en la tradición marcial iraní debido a su alcance, su producción relativamente barata y la sencillez del aprendizaje de sus técnicas. Tuvo un papel muy importante en los periodos de bronce y hierro, que continuaría en siglos posteriores. La lanza aparece citada en muchos manuscritos entre el s. X y el s. XIX. Estos manuscritos persas relatan que los guerreros utilizaban la lanza como arma principal en la batalla, destacando el alcance como su principal virtud; únicamente empleaban otras armas cuando perdían la lanza. Aunque habitualmente los manuscritos no nos ofrecen toda la información que nos gustaría obtener sobre las lanzas, su estudio arroja unos notables resultados en cuanto a tipologías de lanzas y sus técnicas de transporte, ataque y defensa. Con toda seguridad futuras investigaciones sobre la presencia de las lanzas en los manuscritos persas descubrirán nuevas tipologías y técnicas de combate.

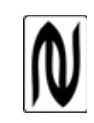

\section{NOTAS}

1 Véase Divān-e Rudaki Samarqandi, del s. X (Rudaki, 2004/1382:91), Aš'ār-e Onsori Balxi, del s. XI (Onsori Balxi, 1990/1369:15), Divān-e Qatrān-e Tabrizi, del s. XI (Qatrān-e Tabrizi, 1983/1362:32) y Masnavi Ma'navi del s. XIII (Molavi, 2006/1385:713). También se utiliza este término en epopeyas como Garšāsbnāme, del s. XI (Asadi Tusi, 1938/1317:50), en manuscritos históricos como Ādāb alHarb va al-Šojā-e, del s. XII (Mobārak Šāh Faxr-e Modabbar, 1967/1346:261) y Tārix-e Ahmad Šăhi del s. XVIII (al-Jāmi, 2001/1379:118) y cuentos de viaje como Safarnāme-ye Nāser Xosrŏ, del s. XI (Nāser Xosrŏ, 1985/1363:74).

2 Para el uso de senān en otros manuscritos véanse los tomos de poemas Aš'ār-e Onsori Balxi, del s. XI (Onsori Balxi, 1990/1369:14), Divān-e Manučehri Dāmqāni, del s. XI (Manučehri Dāmqāni, 1984/1363:9) y Divān-e Qatrān-e Tabrizi del s. XI (Qatrān-e Tabrizi, 1983/1362:15), los cuentos Haft Peykar, del s. XII (Nezāmi Ganje'i, 1999/1377:124) y Xosrŏ va Širin (Nezāmi Ganje'i, 1998a/1376:42), y manuscritos históricos como Tārix-e Jahāngošāy-e Nāderi, del s. XVIII (Esterābādi, 1991/1370:12) y Rostam al Tavārix, del s. XIX (Āsef, 2003/1382:78).

3 Véanse los manuscritos persas Garšāsbnāme, del s. XI (Asadi Tusi, 1938/1317:80) y Ādāb al-Harb va al-Šojā-e, del s. XII (Mobārak Šāh Faxr-e Modabbar, 1967/1346:129).

4 Para el uso de xešt en otros manuscritos véanse las epopeyas Šāhnāme, del s. X (Ferdǒsi, 1995/1384:380), Garšăsbnāme, del s. XI (Asadi Tusi, 1938/1317:485) y Šahanšahnāme, del s. XIX (Sabā, s.f:, 157), los tomos de poemas Divān-e Qatrān-e Tabrizi, del s. XI (Qatrān-e Tabrizi, 1983/1362:35), Divān-e Mas'ud Sa'd Salmān, del s. XI (Sa'd Salmān, 1995/1374:218) y Bustān (Sa'di, 2005/1383:241) y los manuscritos históricos Tārix-e Beihaqi, del s. XI (Beihaqi, 2004/1383:143).

5 Para el uso de zubin en otros mansucritos véanse los manuscritos históricos Tārix-e Beihaqi, del s. XI (Beihaqi, 2004/1383:433) y Ādāb al-Harb va al-Šojā-e, del s. XII (Mobārak Šāh Faxr-e Modabbar (1967/1346:260) y los tomos de poemas Divān-e Qatrān-e Tabrizi, del s. XI (Qatrān-e Tabrizi, 1983/1362:120), Divān-e Mas'ud Sa'd Salmān, del s. XI (Sa'd Salmān, 1995/1374:164) y Bustān (Sa'di, 2005/1383:184). 
6 Para el uso de harbe en otros manuscritos véase Majma' al-Ansāb, del s. XIV (Šabānkāre'i, 2002/1381:181) y los cuentos Haft Peykar (Nezāmi Ganje'i, 1999/1377:24) y Šarafnāme, del s. XII (Nezāmi Ganje'i, 2007/1385a:442).

7 Para el uso de mezrāb veánse los manuscritos Dāstān-e Hossein Kord-e Šabestari, del periodo safávida (Dāstān-e Hossein Kord-e Šabestari, 2003/1382:309), Ālām Ārāye Nāderi (Marvi Vazir Marv, 1985/1374:385) y Rostam al Tavārix, del s. XIX (Āsef, 2003/1382:78, 579). Para el uso de mezrāq véase el tomo de poemas Divān-e Manučehri Dāmqāni, del s. XI (Manučehri Dāmqāni, 1984/1363:48).

8 Véanse el tomo de poemas Divān-e Mas'ud Sa'd Salmān, del s. XI (Sa'd Salmān, 1995/1374:53) y los manuscritos Rostam al Tavārix, del s. XIX(Āsef, 2003/1382:78) y Zafarnāme-ye Xosravi (1999/1377:156).

9 Véanse también los manuscritos Samak Ayyār, del s. XI (al-Kāteb al-Rajāni, 2004/1383:1263) y Divān-e Manučehri Dāmqāni, del s. XI (Manučehri Dāmqāni, 1984/1363:154).

\begin{tabular}{|c|}
\hline $\begin{array}{l}\text { neyze zadan نيزهزن } \\
\text { golpear con la lanza }\end{array}$ \\
\hline $\begin{array}{c}\text { neyze havāle-ye sar kardan نيزه حواله سر كردن } \\
\text { atacar la cabeza con la lanza }\end{array}$ \\
\hline $\begin{array}{c}\text { neyze bar češm zadan نيزه بر جشم زلن } \\
\text { golpear el ojo con la lanza }\end{array}$ \\
\hline $\begin{array}{l}\text { neyze bar galu zadan نيزه بر كلوزدن } \\
\text { golpear el cuello con la lanza }\end{array}$ \\
\hline $\begin{array}{l}\text { neyze dar halq zadan نيزه در حلق زدن } \\
\text { golpear la garganta con la lanza }\end{array}$ \\
\hline $\begin{array}{l}\text { neyze bar dahan zadan نيزه بر دهن زدن } \\
\text { golpear la boca con la lanza }\end{array}$ \\
\hline $\begin{array}{l}\text { neyze bar surat zadan نيزهبر صورت زدن } \\
\text { golpear la cara con la lanza }\end{array}$ \\
\hline $\begin{array}{c}\text { neyze be dast zadan نيزه به دست زدن } \\
\text { golpear la mano con la lanza }\end{array}$ \\
\hline $\begin{array}{c}\text { neyze be sine furu kardan نيزه به سينه فرو كردن } \\
\text { perforar el pecho con la lanza }\end{array}$ \\
\hline $\begin{array}{l}\text { neyze bar šekam zadan نيزه بر شكم زدن } \\
\text { golpear el abdomen con la lanza }\end{array}$ \\
\hline 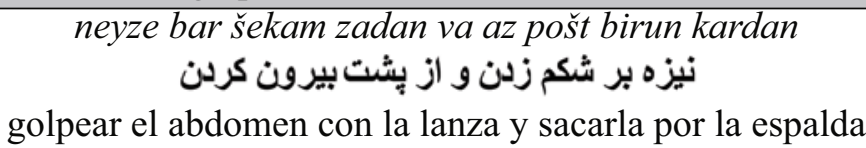 \\
\hline 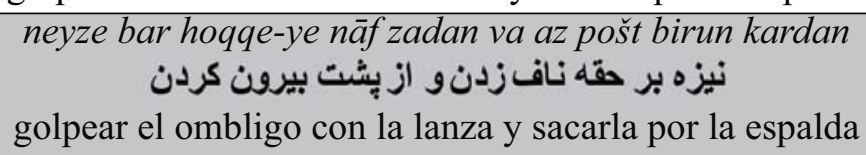 \\
\hline $\begin{array}{l}\text { neyze bar ketf zadan نيزه بر كتف زدند } \\
\text { golpear el hombro con la lanza }\end{array}$ \\
\hline $\begin{array}{c}\text { neyze bar pahlu zadan نيزه بر يهلو زدن (costado) con la lanza } \\
\text { golpear el lado del cuerpo (colo }\end{array}$ \\
\hline
\end{tabular}




\begin{tabular}{|c|}
\hline $\begin{array}{l}\text { neyze bar pošt zadan نيزه بر يشت زدن } \\
\text { golpear la espalda con la lanza }\end{array}$ \\
\hline $\begin{array}{l}\text { neyze bar tohigāh zadan نيزه بر تهيكاهز زن } \\
\text { atacar la region inguinal con la lanza }\end{array}$ \\
\hline $\begin{array}{l}\text { bon-e neyze zadan: بن نيزهزدن } \\
\text { golpear con la contera de la lanza }\end{array}$ \\
\hline $\begin{array}{c}\text { band-e zereh rā boridan بندزرهر ابريدن } \\
\text { cortar las cuerdas de la armadura }\end{array}$ \\
\hline 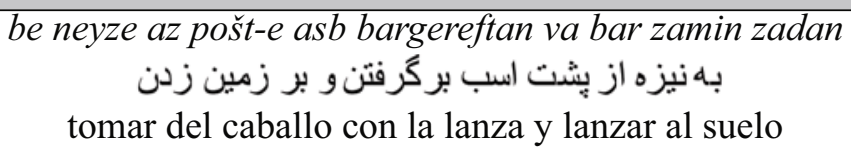 \\
\hline $\begin{array}{l}\text { az zin robudan از زين ربودن } \\
\text { robar de la silla de montar }\end{array}$ \\
\hline
\end{tabular}

\section{Tabla 2: Técnicas de finta con la lanza}

neyze az čap nešān dādan va az rāst bar band-e dast zadan

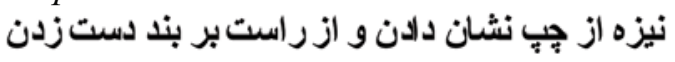

mostrar la lanza a la izquierda y golpear la mano a la derecha

neyze čap nemudan va az rāst be zir-e neyze zadan

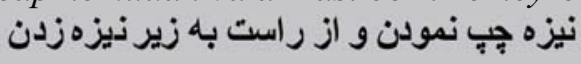

mostrar la lanza a la izquierda y golpear el asta de la lanza desde abajo neyze rā gardāndan va ta'n neyze bar kamargāh zadan

$$
\text { نيزه را كرداندن و طعن نيز بر بر كمركاه زدن }
$$

girar la lanza y golpear la espalda con la punta de la lanza

\section{Tabla 3: Técnicas de ataque al animal con la lanza}

neyze bar pišāni-ye markab zadan نيزه بر بيشانى مركبز زدن golpear la frente del caballo con la lanza

neyze bar sine-ye asb rāndan نيزه بر سينه اسبر اندن golpear el pecho del caballo con la lanza

neyze bar pišāni-ye pil zadan نيزه بر بيشانى بيّل زلدن atacar la frente del elefante con la lanza

\section{Tabla 4: Técnicas de defensa con la lanza}

be čub neyze rā rad kardan جهوب نيزه رارد كردن evadir con el palo [asta]

neyze bar zir-e neyze zadan نيزه بر زير نيزه زدن

golpear la lanza bajo la lanza

neyze az kafrobudan نيزه از كف ربودن

robar la lanza de la mano

neyze rā az kaf birun āvardan نيزهر ا از كف بيرون آوردن

sacar la lanza de la palma de la mano 


\begin{tabular}{|c|}
\hline $\begin{array}{l}\text { neyze rā gereftan نيزهرا كرفنت } \\
\text { agarrar la lanza }\end{array}$ \\
\hline 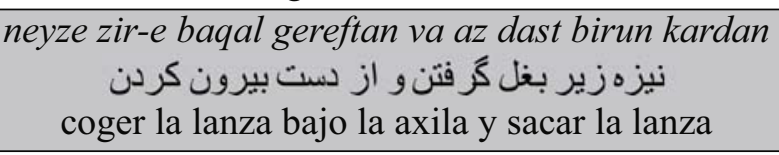 \\
\hline $\begin{array}{l}\text { neyze rā be neyze zadan نيزه را به نيزهزدن } \\
\text { golpear la lanza contra la lanza }\end{array}$ \\
\hline $\begin{array}{c}\text { neyze bar neyze andāxtan نيزه بر نيزه انداختن } \\
\text { meter la lanza sobre la lanza }\end{array}$ \\
\hline
\end{tabular}

\section{Primaria}

\section{BIBLIOGRAFÍA}

ĀSEF, Mohammad HāšEM (RoSTAM Al HoKAMĀ) (2003/1382). Rostam al Tavārix: Salatin-e Selseleye Safaviye, Afšāriye, Zandiye va Qājāriye. [Rostam al Tavārix: Los reyes Safávida, Afs̄ārid, Zand y Qājār]. Comentado por Azizollah Alizādeh. Tehrān: Entešārāt-e Ferdos.

AL-JĀMI, MAHMUd AL-HoSSEINI AL-MonŠI IBN EBRĀHIM (2001/1379). Tārix-e Ahmad Šăhi [Historia de Ahmad Šăh]. Editado y comentado por Puhānd Doktur Sorur Homāyun. Pašāvar: Dāneš Xaparanduye Tulne.

ASADI TuSI, HAKIM ABU NASR-E Ali BEN AHMAD (1938/1317). Garšāsbnāme [La letra/libro de Garšāasb]. Comentado por Yaqmāi. Tehrān: Ketābfuruši va Čāpxāne Baruxim.

AtTĀr NeišĀBuri, AbU HAMID BEN ABU BAKR EbrĀHIM FARID AD-DiN MOHAMMAD (1993/1372). Manteq al-Teyr [Conferencia de las aves]. Anotado por Seyyed Sadeq Goharin. Tehrān: Šerkat-e Entešārāt-e Elmi va Farhangi.

Beihaqi Dabir, Abolfazl Mohammad Ibn Hossein (2004/1383). Tārix-e Beihaqi [Historia de Beihaqi]. Comentado por Ali Akbar Fayyaz. Mašhad: Danešgāh Ferdǒsi Mašhad.

Beiqami, Molānā Mohammad (2002/1381). Dārābnāme [La letra/libro de Dārāb]. Corregido y comentado por Zabiollāh Safā. 2 volúmenes. Tehrān: Šerkat-e Entešārāte Elmi va Farhangi.

ESTERĀBĀDI, MIRZĀ MOHAMMAD MEHDI (1991/1370). Tārix-e Jahāngošāy-e Nāderi [Historia de la conquista del mundo de Nāder]. Manuscrito ilustrado de 1757. Introducción por Abdolali Adib Barumand. Tehrān: Soruš.

FERDǑSI, HAKIM ABOLQĀSEM (1995/1384). Šāhnāme Ferdŏsi (bar Asās-e Nosxeye Mašhur-e be Čap-pe Mosko) [Letra/libro de reyes de Ferdŏsi (basado en la versión de un manuscrito publicado en Moscú)]. Tehrān: Entešārāt-e Alam.

HAKIM, MANOUCHEHR XĀN (s.f.). Koliyat-e Haft Jeldi Eskandarnāme: Sahebqarān Zowalgarenein Eskandar ben Dārāb ben Bahman ben Esfandiār Ruintan [Los siete volumenes completos de la letra/libro de Eskandar: Sahebqarān Zowalgarenein Alexander, el hijo de Dārāb, el hijo de Bahman, el hijo del Invulnerable Esfandiār]. De la serie de leyendas de Pahlavān oriental. Tehrān: Entešārāt-e Mohammad Hasan Sar Elmi.

Kufi, ABu HafAZ (2001/1380). Joneydnāme [Letra/libro de Joneyd]. En: Abu Moslemnāme. Comentado por Hossein Esmā'ili, pp. 199-519. Tehrān: Entešārāt-e Moi’n, Našr-e Qatre, Anjoman-e Irānšenāsi dar Irān. 
MANuČEhri DĀMQĀNi, Abolnajm AhMAd Ben Go's Ben AhMAd (1984/1363). Divān-e Manučehri Dāmqāni [Tomo de poemas de Manučehri Dāmqāni]. Comentado por Mohammd Dabir Siyāqi. Tehrān: Čāp-e Golšan.

MoBĀRAK ŠĀHFAXR-E MODABBAR, MOHAMMAD BENMANSUR BEN SAID (1967/1346). $\bar{A} d \bar{a} b$ al-Harb va al-al-Šojā-e [Costumbres de guerra y coraje]. Comentado por Ahmad Soheili Xānsari. Tehrān: Eqbāl.

Molavi, MŏlānĀ JalāL-AD-Din Mohommad Rumi (2007/1385). Masnavi Ma'navi [El Verso que rima con el que le sigue con un sentido profundo]. Comentado por Reynold A. Nicholson. Tehrān: Entešārāt Hermes.

NĀDERI, AMIR AL-ŠOARĀ (1968/1346). Šāhnāme-ye Nāderi [Letra/libro de reyes de Nāder]. Introducción por Mahmud Farrox. Tehrān: Entešārāte. Ketābxāneye Melli Mālek.

NĀSER XosRŏ QobĀDIYĀNI, ABU Mo'In HAMID AD-DIN (1985/1363). Safarnāme-ye Nāser Xosrŏ [El viaje de Nāser Xosrŏ]. Anotado por Mohammad Dabir Siyāqi. Tehrān: Ketābforuši Zavvār.

NeZĀMI Ganje’', NeZĀM OL-Din AbU Mohammad IlyĀs EBN-E YusOF EBN-E ZĀKI EBN-E MOAYYeD (2007/1385a). Šarafnāme [Letra/libro de reyes de Šaraf]. Comentado por Hasan Vahid Dastgardi. Introducción por Sa'id Hamidiyān. Tehrān: Našr-e Afkār.

NeZĀMI Ganje'I, NeZĀM OL-Din AbU Mohammad IlyĀS EBN-E Yusof EBN-E ZĀKI EBN-E MOAYYed (1999/1377). Haft Peykar [Siete bellezas]. Comentado por Hasan Vahid Dastgardi. Introducción por Sa'id Hamidiyān. Tehrān: Našr-e Qatre.

NeZĀmi Ganje'I, NeZĀm Ol-Din ABu Mohammad IlyāS eBN-E Yusof EBN-E ZĀKI EBN-E MoAYYed (1998/1376). Xosrŏ va Širin [Xosrŏ y Širin]. Anotado por Hasan Vahid Dastgardi. Introducción por Sa'id Hamidiyān. Tehrān: Našr-e Qatre.

OnSORI BALXI, ABOLQĀSEM HASAN BEN AHMAD (1990/1369). Aš ’ār-e Onsori Balxi [Poemas de Onsori Balxi]. Introducción por Mohammad Dabir Siyāqi. Tehrān: Entešārāt-e Amir Kabir.

QATrĀN-E TABrizI, ABU-Mansur (1983/1362). Divān-e Qatrān-e Tabrizi [Poemas de Qatrān-e Tabrizi]. Basado en un manuscrito de Mohammad Naxjavāni. Tehrān: Entešārāt-e Qoqnŏs.

QAZVInI EsfahāNI, Mohammad Yusof VĀLeH (2003/1382). Xold-e Barin: Hadise Šišom va Haftom az Rozeye Haštom [Xold-I Barin (Partes 6, 7, Sección 8)]. Anotado por M.R. Nasiri. Tehrān: Anjoman-e Āsār va Mafāxer Farhangi.

RUdAKi, ABDOlLĀH JAFAR BEN MOHAMmad (2004/1382). Divān-e Rudaki Samarqandi [Tomo de poemas de Rudaki Samarqandi]. Basado en la copia de Sa'id Nafisi. Tehrān: Entešārāt-e Negāh.

SA'D SAlmān, MAS'Ud (1995/1374). Divān-e Mas'ud Sa'd Salmān [Tomo de poemas de Mas 'ud Sa'd Salmān]. Comentado por Rašid Yāsemi. Tehrān: Mo'assese-ye Entešārāte-e Negāh.

SA'di, Moslehodin Mošref Ben ABdollāH (2005/1383). Koliyāt-e Sa'di [Obras completas de Sa'di]. Comentado por Hasan Anvari. Tehrān: Našr-e Qatre.

SABĀ, FATHALI XĀN KĀŠĀNI. Šahanšahnāme [Letrallibro del rey de reyes]. Manuscrito. Mashad: Astān-e Qods-e Razavi.

ŠAbānkĀre'I, Mohammad Ben Ali Ben Mohammad (2002/1381). Majma' alAnsāb (Nimeye Avval) [Majma' al-Ansāb (Primera Parte)]. Comentado por Mir Hāšem Mohaddes. Tehrān: Amir Kabir. 
ŠIRUYE NĀMDĀR (2005/1384). Tehrān: Entešāāāt-e Qoqnŏs.

TAFLISI, HobEYŠ BEN EBRĀHIM BEN MOHAMMAD (1975/1354). Bayān al-Sanā' āt [Explicación de industrías]. Farhang-e Irān Zamin. Volumen 5, segunda edición.

TARTUSI, ABU TĀHER (2001/1380). Abu Moslemnāme [Letra/libro de Abu Moslem]. Comentado por Hossein Esmā'ili. 4 volúmenes. Tehrān: Entešārāt-e Moi’n, Našr-e Qatre, Anjoman-e Irānšenāsi dar Irān.

Tarsusi, Abu TĀHer Mohammad ibn Hasan in Ali iBn Musā (1977/2536). Dārābnāme-ye Tarsusi [La Letra/El Libro de Dārāb escrito por Tarsusi]. Anotado y corregido por Zabihollah Safa. Tehrān. Bongāh-e Tarjome va Našr-e Ketāb.

YAZDI, MolĀNĀ ŠARAF AL-Din Ali (1957/1336). Zafarnāme (Tārix-e Ūmūmi Irān dar Doreyē Teimūriyān) [Letra/libro de victoria (Historia general de Irán durante el periodo Timúrida]. Corregido y comentado por Mohammad Abbāsi. Tehrān: Amir Kabir.

ZAFARNĀME-YE XOSRAVI (1999/1377). Šarh-e Hokmrāvāi-ye Amir Nasrollāh-e Bahādor Soltān ben Heydar (1242-1277 Hégira) dar Boxārā va Samarqand [La historia del dominio de Amir Nasrollāh-e Bahādor Soltān ben Heydar (12421277 Hégira 1827-1860 C.E.) en Buxara y Samarkand]. Escrito anónimo. Anotado por Manučehr Sotude. Tehrān: Daftar Našr-e Mirās-e Maktub.

\section{Secundaria}

BAHRĀMI, EHSĀN AND FEREYDUN JONEYDI (1990/1369). Farhang-e Vājehāye Avestā: Bar Pāye-ye Farhang Kāngā ve Negāreš be Farhanghāye Digar [Lexicón de los términos del Avestā: Basada en el lexicon de Kanga y una reseña de otros lexicones]. Tehrān: Našr-e Balx.

FARAHVAŠI, BAHRĀM (2002a/1381). Farhang Zaban Pahlavi [Lexicón de la lengua pahlavi]. Tehrān: Entešārāt-e Danešgāh-e Tehrān.

FARAHVAŠI, BAHRĀM (2002b/1381). Farhang Farsi be Pahlavi [Lexicón de la lengua persa en pahlavi]. Tehrān: Entešārāt-e Dānešgāh Tehrān.

FARroKh, KAVEH (2005). Sassanian Eilte Cavalry AD 224-642. Oxford: Osprey Publishing Ltd.

MA'TUfi, ASSAdOLlāH (1999/1378). Tārix-e Čāhr Hezār Sāle-ye Arteš Irān: Az Tamaddon-e Ilām Tā 1320 Xoršidi, Jang-e Irān Va Arāq [Cuatro mil años de historia militar iraní: Desde la civilización elámita hasta el año 1320, la Guerra entre Irán y Irak]. Dos vólumenes. Tehrān: Entešārāt-e Imān.

PUR DĀVUd, EBrĀHIM (1969/1347). Zin Abzār (Armamento). Tehrān: Čāpxāne-ye Arteše Šāhanšāhi.

SeKunda, Nicholas (1992). The Persian Army: 560-330 BC. Oxford: Osprey Publishing Ltd.

Wilcox, Peter (2000). Rome's Enemies (3). Oxford: Osprey Publishing.

ZoKĀ, YAHYĀ (1971/1350). Arteše Šāhanšāhi Iran Az Kuroš Ta Pahlavi [El ejército real desde Kurosh hasta Phalavi]. Tehrān: Čāpxāne-ye Vezārat-e Farhang va Honar.

Revista de Artes Marciales Asiáticas $\diamond$ Volumen 4 Número 4 (70-85) - 2009 